\title{
Energy transport in disordered classical spin chains
}

\author{
Vadim Oganesyan* \\ Department of Engineering Science and Physics, College of Staten Island, CUNY, Staten Island, New York 10314, USA
}

\author{
Arijeet $\mathrm{Pal}^{\dagger}$ and David A. Huse \\ Department of Physics, Princeton University, Princeton, New Jersey 08544, USA
}

(Received 27 May 2009; published 3 September 2009)

\begin{abstract}
We present a numerical study of the diffusion of energy at high temperature in strongly disordered chains of interacting classical spins evolving deterministically. We find that quenched randomness strongly suppresses transport with the diffusion constant becoming reduced by several orders of magnitude upon the introduction of moderate disorder. We have also looked for but not found signs of a classical many-body localization transition at any nonzero strength of the spin-spin interactions.
\end{abstract}

DOI: 10.1103/PhysRevB.80.115104

PACS number(s): $71.30 .+\mathrm{h}$

\section{INTRODUCTION}

Chemical, structural, and other imperfections are unavoidable in a typical crystalline solid. Often, reducing their concentration reveals interesting intrinsic properties of the ideal "clean" material. It has long been recognized, however, that sufficiently strong disorder can lead to a host of phenomena essentially distinct from what is observed in clean materials. Anderson localization ${ }^{1}$ is perhaps the best known example of such a phenomenon, whereby a quantum particle becomes coherently trapped by the impurity potential and no longer participates in transport. Essentially a wave phenomenon, Anderson localization can also occur for classical linear waves, e.g., photons or phonons.

It has often been assumed that true localization (strictly zero diffusivity) of interacting particles can only happen at absolute zero temperature, even though Anderson's original paper on localization discusses the possibility of localization persisting at nonzero temperature. ${ }^{1}$ Recently this question has been examined carefully by Basko et al., ${ }^{2}$ who performed a stability analysis of an Anderson insulator against weak interparticle interactions at low but nonzero temperature. Their central conclusion is that an isolated system of strongly disordered but weakly interacting quantum particles should exhibit a transition into an insulating phase with strictly zero diffusion at some low but nonzero excitation energy per particle (or temperature). Motivated by this work, two of us $^{3}$ considered quantum lattice models that would be expected to exhibit such a dynamical many-body localization transition as one varies the interactions or disorder strength, even at arbitrarily high temperature. We attempted to detect this transition by studying exact many-body spectral statistics of small lattices. Initial results are encouraging, ${ }^{3}$ although they fall short of making a strong case for the existence of a quantum many-body localization transition, due to strong finite-size effects.

Setting aside the question of the existence of such a transition (i.e., assuming it does exist), one might wonder about its nature, e.g., the universality class. On the one hand, the theoretical analysis of Basko et al. ${ }^{2}$ relies entirely on quantum many-body perturbation theory. Rather generally, however, one expects macroscopic equilibrium and lowfrequency dynamic properties of interacting quantum systems at nonzero temperature to be describable in terms of effective classical models. This expectation is certainly borne out in a variety of symmetry-breaking phase transitions with a diverging correlation length, such as, e.g., a finite temperature Néel ordering of spin-1/2 moments. One can begin to understand the microscopic mechanism behind such a manybody "correspondence principle" as a consequence of an effective coarse graining, whereby the relevant degrees of freedom are correlated spins moving together in patches that grow in size as the phase transition is approached and therefore become "heavy" and progressively more classical. Further extension of these ideas to general, noncritical, dynamical response is more involved: roughly speaking, it requires that the typical many-body level spacing in each patch be much smaller than the typical matrix element of interactions with other patches. If this is true (as it is in most models at finite temperature, though not necessarily in the insulating phase analyzed by Basko and collaborators) one replaces microscopic quantum degrees of freedom with macroscopic classical ones, which typically obey "hydrodynamic" equations of motion at low frequencies. ${ }^{4}$ Since it is expected that the many-body localization transition is accompanied by a diverging correlation length (akin to the Anderson transition) one might expect some sort of classical description to emerge enroute from the localized phase to the diffusive phase. It was this thinking that initially motivated us to consider the possibility of classical many-body localization.

The process by which collective classical (hydro) dynamics emerges from a microscopic quantum description is subtle and may or may not be relevant to the many-body localization discussed above. A somewhat less subtle but apparently largely unexplored related question is whether nonlinear, interacting, and disordered classical many-body systems are capable of localization at nonzero temperature. To be precise, a many-body classical dynamical system with a local Hamiltonian (including static randomness) should show hydrodynamic behavior, e.g., energy diffusion, provided the local degrees of freedom are nonlinear and interacting, and the disorder is not too strong. In this regime, the isolated system can function as its own heat bath and relax to thermal equilibrium. Diffusive energy transport must stop if the interactions between the local degrees of freedom are turned off. How is this limit approached? Can there be a classical 
many-body localization transition where the energy diffusivity vanishes while the interactions remain nonzero? These are the basic questions we set out to investigate in this paper.

Our preliminary conclusion is that classical many-body systems with quenched randomness and nonzero nonlinear interactions do generically equilibrate, so there is no generic classical many-body localized phase. Our picture of why this is true is that generically a nonzero fraction of the nonlinearly interacting classical degrees of freedom are chaotic and thus generate a broadband continuous spectrum of noise. This allows them to couple to and exchange energy with any other nearby degrees of freedom, thus functioning as a local heat bath. Random classical many-body systems generically have a nonzero density of such locally chaotic "clusters" and thus the transport of energy between them is over a finite distance and cannot be strictly zero, resulting in a nonzero (although perhaps exponentially small) thermal conductivity. Quantum systems, on the other hand, cannot have a finite cluster with a truly continuous density of states: the spectrum of a finite cluster is always discrete. Thus the mechanism that we propose forbids a generic classical many-body localized phase, yet it does not appear to apply to the quantum case. The proposed existence of the many-body insulator in quantum problems is then a remarkable manifestation of quantum physics in the macroscopic dynamics of highly excited matter. In this paper we shall primarily focus on macroscopic low-frequency behavior, postponing detailed analysis of local structure of noise and its relation to transport. Our conclusions are broadly consistent with findings of Dhar and Lebowitz ${ }^{5}$ although given the rather major differences in models, methods, and, most importantly, the extent to which strongly localized regime is probed we refrain from making direct comparisons.

We study energy transport in a simple model of local many-body Hamiltonian dynamics that has both strong static disorder and interactions: classical Heisenberg spin chains with quenched random fields. We study a one-dimensional model both because one dimension is more accessible to numerical study and because we are looking for localization, which should be most likely in this the lowest of nonzero dimensions. For simplicity, we consider the limit of infinite temperature, defined by averaging over all initial conditions with equal weights. Our systems conserve the total energy and should exhibit energy diffusion; they have no other conservation laws. The energy diffusion coefficient, $D$, can be deduced from the autocorrelations of the energy current (as explained below) and is shown in Fig. 1 as a function of the strength of the spin-spin interactions, $J$. The mean-square random field is $\Delta^{2}$, and as we vary $J$ we keep $2 J^{2}+\Delta^{2}=1$, as explained below. The limit $J \rightarrow 0$ is where the interactions vanish, so there is (trivially) no energy transport.

As the interaction $J$ is decreased, the thermal diffusivity $D$ decreases very strongly; we have been able to follow this decrease in $D$ for about five orders of magnitude before the systems' dynamics become too slow for our numerical studies. For most of this range, we can roughly fit $D(J)$ with a power law, $D \sim J^{\gamma}$, with a rather large exponent, $\gamma \cong 8$, as illustrated in Fig. 1. This large exponent suggests that the asymptotic behavior at small interaction $J$ may be some sort of exponential, rather than power law, behavior, consistent

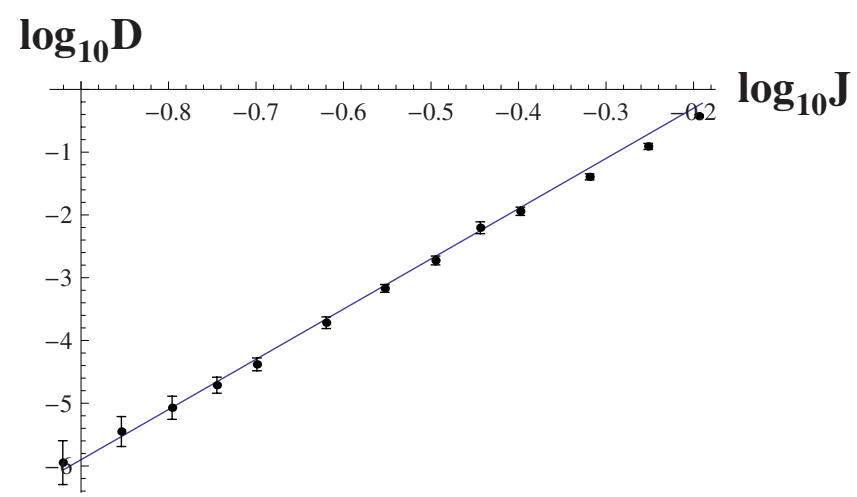

FIG. 1. (Color online) Disorder-averaged energy diffusion constant $D$ as a function of the spin-spin interaction $J$. The line has slope 8 on this log-log plot.

with the possibility that the transport is actually essentially nonperturbative in $J$. In principle, it is also possible to fit these data to a form with a nonzero critical $J_{c}$ so that $D(J$ $\left.<J_{c}\right)=0$ - such fits prove inconclusive as they produce estimates of $J_{c}$ considerably smaller than the values of $J$ where we can measure a nonzero $D$. Since we are not aware of any solid theory for the behavior of $D(J)$, these attempts at fitting the data are at best suggestive. The large range of variation in the macroscopic diffusion constant $D$ across a rather modest range of $J$ is the most clearly remarkable and robust finding that we wish to present in this paper.

Our model and general methods employed will be presented and discussed in the next section. Much of what we present is based on the analysis of energy current fluctuations in isolated rings. For various reasons we have found it beneficial to focus on these rather than fluctuations of the energy density or on current carrying states in open systems (we have spot checked for quantitative agreement among these three methods). In Sec. III we present our results for macroscopic transport starting from short-time behavior that is relatively easy to understand and working up to long time, dc behavior that is both difficult to compute and as of yet poorly understood. One particularly interesting observation we make here is that of a subdiffusive behavior at long times, apparently distinct from the much discussed mode-coupling behavior well representative of linear diffusion in the presence of disorder. We discuss some afterthoughts and open problems in the summary with some important additional details relegated to Appendices A and B (such as quantitative explorations of finite-size effects, roundoff, many-body chaos, and self-averaging).

\section{MODEL, TRAJECTORIES, AND TRANSPORT}

The classical motion of $N$ interacting particles is usually defined by a system of coupled differential equations of motion. The "particles" we study here are classical Heisenberg spins-three-component unit-length vectors, $\mathbf{S}_{i}$, placed at each site $i$ of a one-dimensional lattice. With a standard angular momentum Poisson bracket and a Hamiltonian, $H$, the equations of motion are 


$$
\frac{\partial \mathbf{S}_{j}}{\partial t}=\mathbf{H}_{j} \times \mathbf{S}_{j}
$$

where $\mathbf{H}_{j}=\partial H / \partial \mathbf{S}_{j}$ is the total instantaneous field acting on spin $\mathbf{S}_{j}$. The Hamiltonians we consider are all of the form

$$
H=\sum_{j}\left(\mathbf{h}_{j} \cdot \mathbf{S}_{j}+J \mathbf{S}_{j} \cdot \mathbf{S}_{j+1}\right)
$$

with uniform pairwise interaction $J$ between nearestneighbor spins and quenched random magnetic fields, $\mathbf{h}_{j}$. For almost all of the results in this paper, we choose the random fields to be $\mathbf{h}_{j}=h_{j} \hat{\mathbf{n}}_{j}$, where the $h_{j}$ are independent Gaussian random numbers with mean zero and variance $\Delta^{2}$ while the $\hat{\mathbf{n}}_{j}$ are independent randomly oriented unit vectors, uniformly distributed in orientation. Because of the random fields, total spin is not conserved and we can focus on energy diffusion as the only measure of transport in this system. For $J=0$ and $\Delta>0$ any initial distribution of energy is localized, as the spins simply precess indefinitely about their local random fields, so the diffusivity is $D=0$. In the opposite limit, where $\Delta=0$ and $J>0$, there is diffusive transport with $D \sim J$ (with nonlinear corrections due to the coupling between energy and spin diffusion $\left.{ }^{4,6}\right)$. We are interested in the behavior of $D$ as one moves between these two limits, especially as one approaches $J=0$ with $\Delta>0$.

Given initial spin orientations, it is in principle straightforward to integrate the equations of motion numerically, thus producing an approximate many-body trajectory. Correlation functions can then be computed and averaged over such trajectories and realizations of the quenched random fields. The transport coefficients can thereby be estimated via the fluctuation-dissipation relations.

\section{A. Model}

Before we embark on this program, however, we start by making a change to the model's dynamics [Eq. (1)] but not to its Hamiltonian (2), in order to facilitate the numerical investigation of the long-time regime of interest to us, where the diffusion is very slow. In order to get to long times with as little computer time as possible, we want our basic time step to be as long as is possible. What we are interested in is not necessarily the precise behavior of any specific model but the behavior of the energy transport in a convenient model of the type (2). Since we are studying energy transport, it is absolutely essential that the numerical procedure we use does conserve total energy (to numerical precision) and that the interactions and constraints remain local. Thus we modify the model's dynamics to allow a large time step while still strictly conserving total energy.

We change the equations of motion (1) of our model so that the even- and odd-numbered spins take turns precessing, instead of precessing simultaneously. We will usually have periodic boundary conditions, so we thus restrict ourselves to even length (thus bipartite) chains. We use our basic numerical time step as the unit of time (and the lattice spacing as the unit of length). During one time step, first the odd-numbered spins are held stationary while the even-numbered spins precess about their instantaneous local fields,

$$
\mathbf{H}_{r}(t)=\mathbf{h}_{r}+J \mathbf{S}_{r-1}(t)+J \mathbf{S}_{r+1}(t),
$$

by the amount they should in one unit of time according to Eq. (1). Note that since the odd spins are stationary, these local fields on the even sites are not changing while the even spins precess so that this precession can be simply and exactly calculated, and the total energy is not changed by this precession. Then the even spins are stopped and held stationary in their new orientations while the odd spins "take their turn" precessing to complete a full time step. Although this change in the model's dynamics from a continuous-time evolution to a discrete-time map is substantial, we do not expect it to affect the qualitative long-time, low-frequency behavior of the model that is our focus in this paper. In particular, we clearly observe correct diffusive decay of local correlations for weak disorder and essentially indefinite precession of spins at very strong disorder.

We have decided to use parameters so that the meansquare angle of precession of a spin during one time step is one radian (at infinite temperature), which seems about as large as one can make the time step and still be roughly approximating continuous spin precession. This choice dictates that the parameters satisfy

$$
2 J^{2}+\Delta^{2}=1 .
$$

We will generally describe a degree of interaction by quoting the $J$; the strength $\Delta$ of the random field varies with $J$ as dictated by Eq. (4).

\section{B. Observables}

The basic observable of interest, the instantaneous energy $e_{i}(t)$ at site $i$ is

$$
e_{i}(t)=\mathbf{h}_{i} \cdot \mathbf{S}_{i}(t)+\frac{J}{2}\left[\mathbf{S}_{i-1}(t) \cdot \mathbf{S}_{i}(t)+\mathbf{S}_{i+1}(t) \cdot \mathbf{S}_{i}(t)\right] .
$$

Note that with this definition, the interaction energy corresponding to a given bond is split equally between the two adjacent sites. When updating the spin at site $i$, only the energies of the three adjacent sites, $e_{i}$ and $e_{i \pm 1}$, change due to the change in the interaction energies involving spin $i$. This rather simple pattern of rearrangement of energy allows for an unambiguous definition of the energy current at site $i$ during the time step from time $t$ to $t+1$. If site $i$ is even, so it precesses first, then the current is

$$
j_{i}(t)=J\left[\mathbf{S}_{i}(t+1)-\mathbf{S}_{i}(t)\right] \cdot\left[\mathbf{S}_{i+1}(t)-\mathbf{S}_{i-1}(t)\right]
$$

while for $i$ odd,

$$
j_{i}(t)=J\left[\mathbf{S}_{i}(t+1)-\mathbf{S}_{i}(t)\right] \cdot\left[\mathbf{S}_{i+1}(t+1)-\mathbf{S}_{i-1}(t+1)\right] .
$$

We are working at infinite temperature or alternatively at $\beta=\left(k_{B} T\right)^{-1}=0$. The conventionally defined thermal conductivity vanishes for $\beta \rightarrow 0 .^{4}$ Instead, here we define the dc thermal conductivity $\kappa$ so that the average energy current obeys

$$
j=\kappa \nabla \beta
$$

in linear response to a spatially and temporally uniform small gradient in $\beta=1 /\left(k_{B} T\right)$. The Kubo relation then relates this 
thermal conductivity at $\beta=0$ to the correlation function of the energy current via

$$
\kappa=\sum_{t} C(t),
$$

where

$$
C(t)=\sum_{i}\left[\left\langle j_{0}(0) j_{i}(t)\right\rangle\right]
$$

is the autocorrelation function of the total current, where the square brackets, $[\cdots]$, denote a full average over instances of the quenched randomness ("samples") and the angular brackets, $\langle\cdots\rangle$, denote an average over initial conditions in a given sample and time average within a given run. For our model (2) the average energy per site obeys

$$
\frac{d[\langle e\rangle]}{d \beta}=\frac{J^{2}+\Delta^{2}}{3}
$$

at $\beta=0$ and the energy diffusivity $D$ is then obtained from the relation

$$
\frac{\kappa}{D}=\frac{d[\langle e\rangle]}{d \beta} .
$$

In a numerical study, if a quantity (such as $\kappa$ ) is nonnegative definite, then it is helpful to measure it if possible as the square of a real measurable quantity. We use this approach here, noting that

$$
\kappa=\lim _{L, t \rightarrow \infty} \frac{1}{L t}\left[\left\langle\left\{\sum_{\tau=1}^{t} \sum_{i=1}^{L} j_{i}(\tau)\right\}^{2}\right\rangle\right] .
$$

For a particular instance of the random fields in a chain of even length $L$ with periodic boundary conditions and a particular initial condition $I$ run for time $t$, we thus define the resulting estimate of $\kappa$ as

$$
\kappa_{I}(t)=\frac{1}{L t}\left\{\sum_{\tau=1}^{t} \sum_{i=1}^{L} j_{i}(\tau)\right\}^{2} .
$$

If these estimates are then averaged over samples and over initial conditions for a given $L$ and $t$, this results in the estimate $\kappa_{L}(t)=\left[\left\langle\kappa_{I}(t)\right\rangle\right]$. These estimates $\kappa_{L}(t)$ must then converge to the correct dc thermal conductivity $\kappa$ in the limits $L, t \rightarrow \infty$.

\section{Finite-size and finite-time effects}

In a sample of length $L$, we expect finite-size effects to become substantial on time scales

$$
t>t_{L}=C_{D} L^{2} / D_{e f f},
$$

where $D_{\text {eff }}$ is the effective diffusion constant at those time and length scales, and we find $C_{D} \cong 10$ [remarkably Eq. (15) remains valid more or less with the same value of $C_{D}$ across the entire range of parameters-see Appendix A]. With periodic boundary conditions (which is the case in our simulations) this means that $\kappa_{L}(t)$ saturates for $t>t_{L}$ to a value different from (and usually above) its true dc value in the infinite $L$ limit while with open boundary conditions (no energy transport past the ends of the chain) the infinite-time limit of $\kappa_{L}(t)$ is instead identically zero for any finite $L$. We simply avoid this purely hydrodynamic finite-size effect by using chains of large enough length $L$, which is relatively easy, especially in the strongly disordered regime of interest, where $D_{\text {eff }}$ is quite small.

For the smallest values of $J$ that we have studied, the system is essentially a thermal insulator and the $D_{\text {eff }}$ is so small that finite-size effects are just not visible at accessible times even for small values of $L$, such as $L=10$. Instead, given the way we are estimating $\kappa$, a finite-time effect, due to the sharp "cutoffs" in time at time zero and $t$ in Eq. (14), dominates the estimates $\kappa_{L}(t) \sim J^{2} / t$ in this small- $J$ regime. To explain this better we can rewrite the definition of $\kappa$ as

$$
\kappa_{L}(t)=\frac{1}{L t} \sum_{\tau=1}^{t} \sum_{\tau^{\prime}=1}^{t} C\left(\tau-\tau^{\prime}\right)=\frac{2}{L t} \sum_{t_{a v}=1}^{t / 2} \kappa_{L}^{*}\left(t_{a v}\right),
$$

where we have assumed an even $t$ (there is an additional term otherwise) and $\kappa_{L}^{*}(\tau) \equiv \Sigma_{-\tau}^{\tau} C\left(\tau^{\prime}\right)$. Localization, i.e., zero dc conductivity, implies a rapidly vanishing $\kappa^{*}$ as well as $\kappa$ at long times. The latter however acquires a tail, $\kappa \sim 1 / t$ whose amplitude is set by the short-time values of $\kappa^{*}$.

For the intermediate values of $J$ that are of the most interest to us in this paper, there is also another, stronger finitetime effect due to an apparently power-law "long-time tail" in the current autocorrelation function, $C(\tau)$, as we discuss in detail below. Importantly, at long times this intrinsic finitetime effect dominates the extrinsic, cutoff induced, $1 / t$ effect discussed above, so $\kappa_{L}(t)$ remains a useful quantity to study in this regime.

\section{RESULTS: MACROSCOPIC DIFFUSION}

\section{A. Current autocorrelations}

Since the total current is not dynamically stationary, its autocorrelation function, $C(t)$, should decay in time. In a strongly disordered dynamical system we expect the dc conductivity, which is the sum over all times of this autocorrelation function, to be very small due to strong cancellations between different time domains [i.e., $C(t)$ changes sign with varying $t]$. The basic challenge of computing the dc thermal conductivity $\kappa$ boils down to computing (and understanding) this cancellation.

The autocorrelation function $C(t)$ has three notable regimes as we vary $J$ and $t$. First, $C(t)$ is positive and of order $J^{2}$ at times less than or of order 1, as illustrated in Fig. 2. It quickly becomes negative at larger times. For small $J$ it is negative and of order $J^{3}$ in magnitude for times of order $1 / J$ (see Fig. 3). For very small $J$, this negative portion of $C(t)$ almost completely cancels the short-time positive portion, resulting in an extremely small $\kappa^{*}$ (see inset in Fig. 3). This cancellation is a hallmark of strong localization and can be observed, e.g., in an Anderson insulator where it is nearly complete $\left(\kappa^{*} \rightarrow 0\right.$ exponentially with time). While the very short-time behavior at small $J$ is easily reproduced analytically by ignoring dynamical spin-spin correlations, the behavior out to times of order $1 / J$ is representative of corre- 


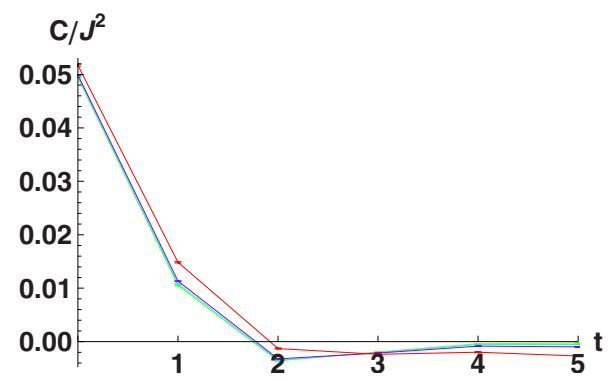

FIG. 2. (Color online) Short-time behavior of $C(t)$ for $J=0.32$ (red, noticeably different trace) and $J=0.08,0.12,0.16$ (these are almost identical data in this plot). Note rescaling of the vertical axis by $J^{2}$.

lated motion of few spins (likely pairs). Although likely nonintegrable, this motion is nevertheless mostly quasiperiodic-we recorded indications of this in local spinspin correlation functions (not shown here).

Finally, there is apparently a power-law long-time tail with a negative amplitude: $C(t) \sim-t^{-1-x}$, with an exponent that we find is approximately $x \cong 0.25$ over an intermediate range of $0.2 \leqq J \leqq 0.4$ (and more generally, perhaps). To observe this with the least amount of effort it is best to average $C(t)$ at long times over a neighborhood of $t$ (see Fig. 4) or to measure $\kappa_{L}(t)$ and compute its "exponential derivative," $\eta(t) \equiv \kappa_{L}(t)-\kappa_{L}(2 t)$, at a sequence of points $t_{n}=2^{n}, n$ $=1,2,3, \ldots$ (see Fig. 5). The apparent value $x \cong 0.25$ of this exponent is something that we do not understand yet theoretically. However, we find that it does provide a good fit to the data over a wide dynamic range, providing some support for our use of it to extrapolate to infinite time and thus estimate the dc thermal conductivity, as discussed below.

\section{B. de conductivity: Extrapolations and fits}

Our extrapolations of the dc conductivity will be based entirely on the long-time behavior of $\kappa_{L}(t)$ evaluated at a set of times $t_{n}=2^{n}$ with integer $n$ and for large enough $L$ to eliminate finite-size effects (so we drop the subscript $L$ ). We

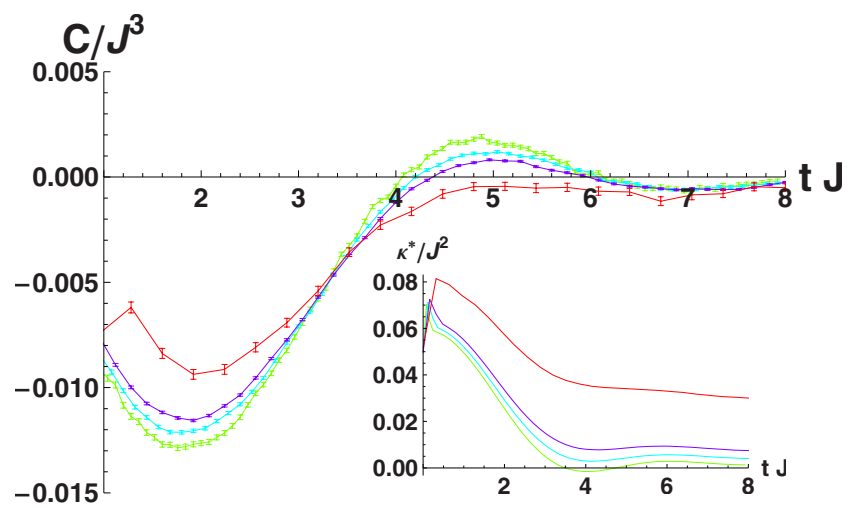

FIG. 3. (Color online) Current autocorrelations on medium time scales $\sim 1 / J$ for $J=0.32,0.16,0.12,0.08$, from top (red) to bottom (green) trace at $t J=2$. Note the rescaling of both the vertical and time axes. The inset shows near cancellation between short and medium times.
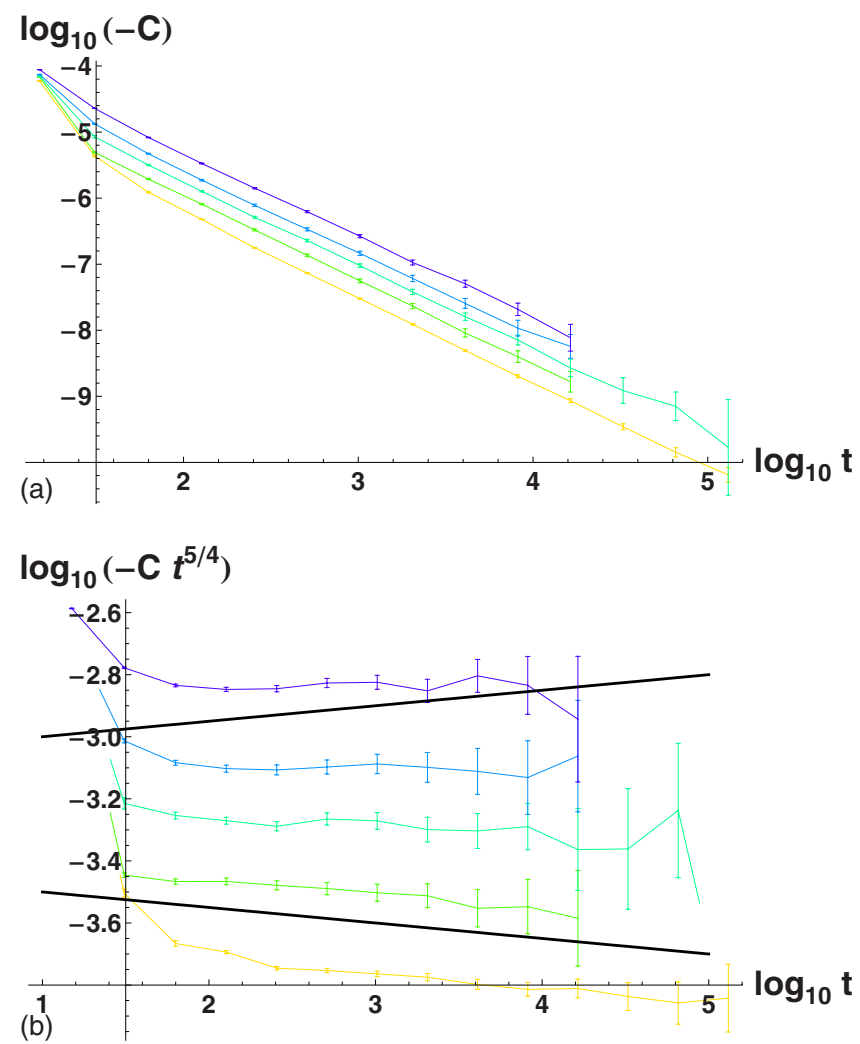

FIG. 4. (Color online) Top panel: long-time tail in the current autocorrelation function for $J=0.20,0.24,0.28,0.32,0.40$ shown from bottom to top in the order of $J$ listed. Bottom panel: to estimate the exponent we multiply the data by $t^{5 / 4}$ (and also display lines with slope \pm 0.05 ). Although these data do not exclude an exponent that varies with $J$, we interpret these results as supportive of a single exponent $x \approx 0.25$ at asymptotically long times but with a more pronounced short-time transient at smaller $J$.

start by describing the procedure used to arrive at the numerical estimates of the dc conductivity, then turn to the subject of uncertainties.

A typical instantaneous value of the energy current is set by the strength of the exchange, $J$. As a consequence $\kappa(t)$

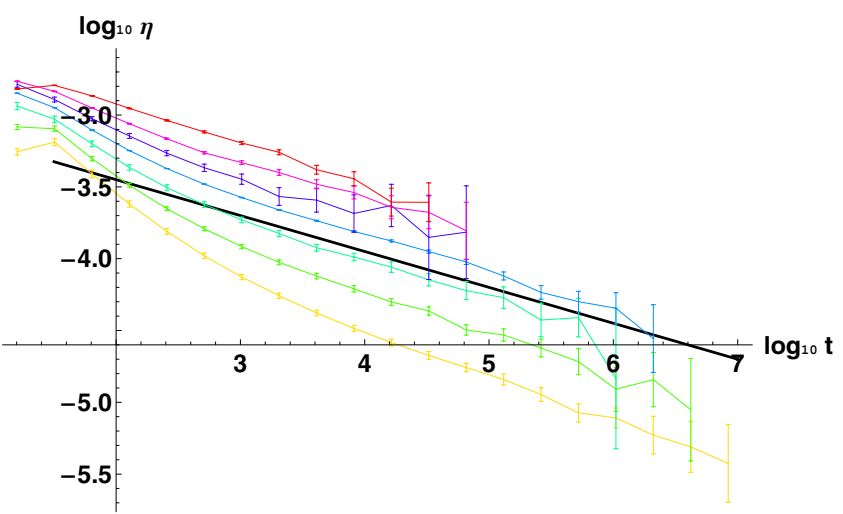

FIG. 5. (Color online) Long time tails as seen from $\eta(t)$ for $J$ $=0.20,0.24,0.28,0.32,0.36,0.40,0.48$ (bottom to top). Black line is a guide to the eye with slope $-1 / 4$. Note that the short-time transients are stronger here, as compared to the autocorrelation data in Fig. 4. 


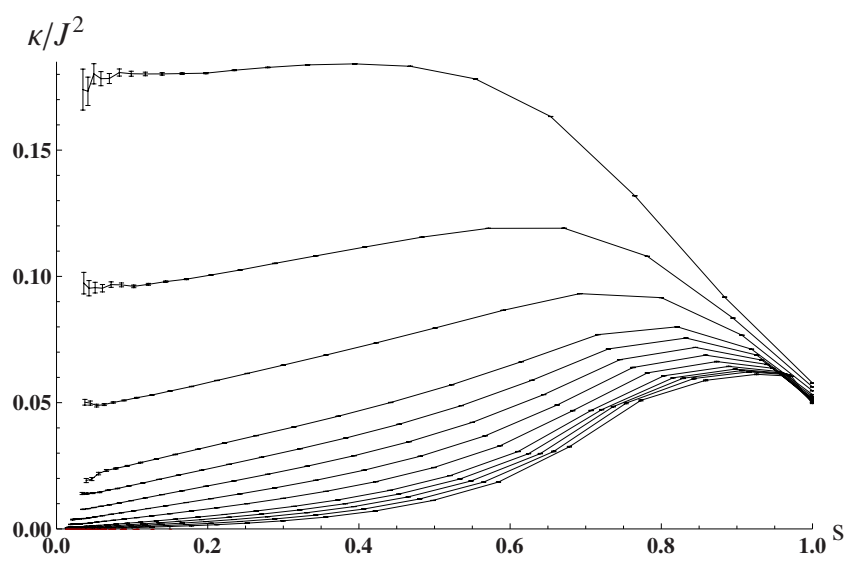

FIG. 6. (Color online) Variation in $\kappa(t)$ for of $J=0.64$, $0.56,0.48,0.40,0.36,0.32,0.28,0.24,0.20,0.18,0.16,0.14,0.12$ plotted vs $s=(1+J t)^{-0.25}$ (larger $J$ have larger $\kappa$ as $\left.s \rightarrow 0\right)$. Lines are merely guides to the eye and statistical errors are too small to be seen on most of these points. This figure is used for obtaining $J$ $\geq 0.36$ entries in Table I.

$\sim J^{2}$ for small $J$ at short and intermediate times ( $t$ of order $1 / J$ or less). Given the time dependence at intermediate and long times, as discussed above, we adopt the variable $s=(1$ $+J t)^{-0.25}$ as a convenient "scaling" of time for displaying our results. These rescalings "collapse" the observed values of $\kappa(t)$ for short to intermediate times across the entire range of $J$ studied, as shown in Fig. 6.

The extrapolated values of the dc conductivity decrease strongly as $J$ is reduced. Extrapolation of $\kappa(t)$ to $s=0$ and thus dc is fairly unambiguous for $J \geq 0.32$, as can be seen in Fig. 6. To display the long-time results at smaller $J$, in Fig. 7 we instead show $\kappa / J^{10}$. Here one can see that as we go to smaller $J$ the extrapolation to the dc limit $(s=0)$ becomes more and more of "a reach" as $J$ is reduced. The outcomes of these extrapolations and rough estimates of the uncertainties are summarized in Table I.

There are several sources of uncertainty in the estimates of the dc thermal conductivity $\kappa$ reported in Table I. These can be separated into those originating with the measured values of $\kappa_{L}(t)$ and those due to the extrapolation to dc.

The statistical uncertainties in the measured values of $\kappa_{L}(t)$ were estimated (and shown in the figures) from sampleto-sample fluctuations which we find follow Gaussian statistics to a good approximation for these long (large $L$ ) samples. We did look for a possible systematic source of error originating with roundoff and its amplification by chaos (see Appendix B) and found it not to be relevant for the values of $J$ and $t$ studied.

The uncertainties in our estimates of the $\mathrm{dc} \kappa$ from the extrapolation procedure begin with the assumed value of the long-time power law, $x \cong 0.25$. Clearly, using a different exponent will change the extrapolated dc values of $\kappa$ somewhat. This uncertainty increases with decreasing $J$ as the ratio of the $\kappa_{L}(t)$ at the last time point to the extrapolated value increases. At our smallest $J$ values, the curvature in our $\kappa$ vs $s$ plots due to the crossover to the earlier-time insulatinglike $\sim 1 / t \sim s^{4}$ dependence becomes more apparent and further complicates the extrapolation. Although we have ex-

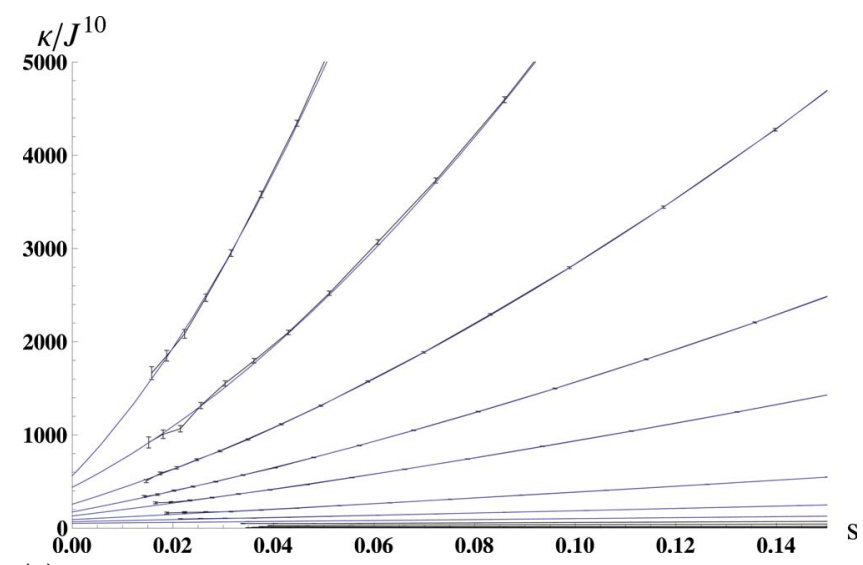

(a)

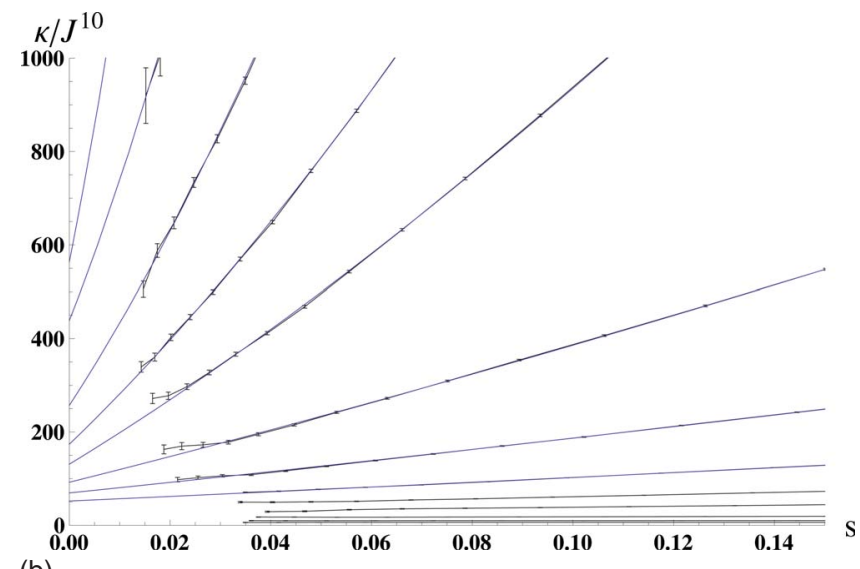

(b)

FIG. 7. (Color online) Same data as in Fig. 6 but now scaled and displayed in a way that allows one to see the extrapolations to $s$ $=0(t \rightarrow \infty)$ for small $J$. Because of the rescaling of the plotted in the previous figure. Note the different rescaling schemes used in preceding and current plots to focus on collapse of short-time data (previous plot) vs long-time extrapolations (present plot). As before black lines are drawn through the data for guiding the eyes. Colored lines are results of polynomial fits, as explained in the text. This figure is used for obtaining entries in Table I for $J \leq 0.32$.

perimented with some different schemes for extrapolating to $\mathrm{dc}$, including different choices of exponent $x$, in the end the following procedure appeared to capture the overall scale of the diffusion constant and with a generous estimate of the uncertainty: (i) we start by removing early data with $s \gtrsim 0.5$ to focus strictly on the long-time behavior; (ii) this long-time dependence is further truncated by removing five latest points and then fitted to a polynomial $\Sigma_{0}^{4} a_{n} s^{n}$ to better capture the curvature apparent in the data-these fits are shown in Fig. 7 and $a_{0}$ are the dc values reported in Table I; and (iii) the uncertainty is estimated as the greater of statistical error in the last point (which is negligible for most of our data) and the difference between $a_{0}$ and a simple linear extrapolation performed on latest five data points not included in (ii).

Overall, we deem the values presented in Table I as "safe" since all extrapolated $\kappa$ 's differ by at most a factor 2 from $\kappa$ 's actually measured, in other words our extrapolations are reasonably conservative (with the exception of two smallest $J$ 's where the extrapolations yield stronger reductions). 
TABLE I. Extrapolated estimates of the dc conductivity $\kappa$, estimated uncertainties, length $L$ of samples, and the number of time steps $T$ of the runs.

\begin{tabular}{cccccc}
\hline \hline$J$ & $\kappa$ & $\delta \kappa$ & $L$ & $\log _{2} T$ & Samples \\
\hline 0.64 & $0.18 J^{2}$ & $0.01 J^{2}$ & 5000 & 20 & 1000 \\
0.56 & $0.09 J^{2}$ & $0.01 J^{2}$ & 2000 & 20 & 1000 \\
0.48 & $0.045 J^{2}$ & $0.005 J^{2}$ & 1000 & 20 & 4000 \\
0.40 & $0.020 J^{2}$ & $0.003 J^{2}$ & 1000 & 20 & 1000 \\
0.36 & $0.014 J^{2}$ & $0.003 J^{2}$ & 1000 & 21 & 2200 \\
0.32 & $50 J^{10}$ & $8 J^{10}$ & 1000 & 21 & 16000 \\
0.28 & $70 J^{10}$ & $10 J^{10}$ & 1000 & 24 & 912 \\
0.24 & $95 J^{10}$ & $20 J^{10}$ & 1000 & 25 & 558 \\
0.20 & $130 J^{10}$ & $30 J^{10}$ & 1000 & 26 & 1179 \\
0.18 & $175 J^{10}$ & $50 J^{10}$ & 500 & 27 & 2000 \\
0.16 & $250 J^{10}$ & $100 J^{10}$ & 500 & 27 & 1550 \\
0.14 & $400 J^{10}$ & $200 J^{10}$ & 500 & 27 & 520 \\
0.12 & $600 J^{10}$ & $400 J^{10}$ & 500 & 27 & 1116 \\
\hline \hline
\end{tabular}

\section{SUMMARY, FURTHER EXPLORATIONS, AND OUTLOOK}

In summary, we considered a rather generic model of classical Hamiltonian many-body dynamics with quenched disorder and explored the systematic variation in the thermal diffusivity between conducting and insulating states. We found a rapid variation in the diffusion constant and presented quantitative estimates of the latter across more than five orders of magnitude of change. The origin of this behavior may be traced to spatial localization of classical few-body chaos-we plan to present further results along these lines separately. Qualitatively, such a scenario is rather plausible at very low $J$, where most spins are spectrally decoupled due to disorder and essentially just undergo independent Larmor precessions. As long as $J$ is nonzero, however, there will always be a fraction of spins in resonance with some of their immediate neighbors. These clusters are then deterministically chaotic and thus generate broadband noise, which allows them to exchange energy with all other nearby spins. Importantly, in the entire parameter range studied this heterogeneous regime eventually gives way at long time to a more homogeneous conducting state in the dc limit. Thus, we suspect that internally generated but localized noise always causes nonzero dc thermal transport even in the strongly disordered regime as long as the spin-spin interaction $J$ is nonzero.

Additionally, we also discovered and characterized an apparent, finite-time (frequency) correction to diffusion, with the diffusivity varying as $D(\omega) \approx D(0)+a|\omega|^{x}$ with $x \cong 0.25$. Previous theoretical work on corrections to diffusion due to quenched disorder ${ }^{7}$ have instead found a correction with exponent $x=1 / 2$, which is quite inconsistent with our numerical results. This power-law behavior is apparently not due to the localization of chaos discussed above, as it persists well into the strongly conducting regime (larger $J$ ) and also exists in models without a strong disorder limit at all (e.g., with random fields of equal magnitude but random direction; data

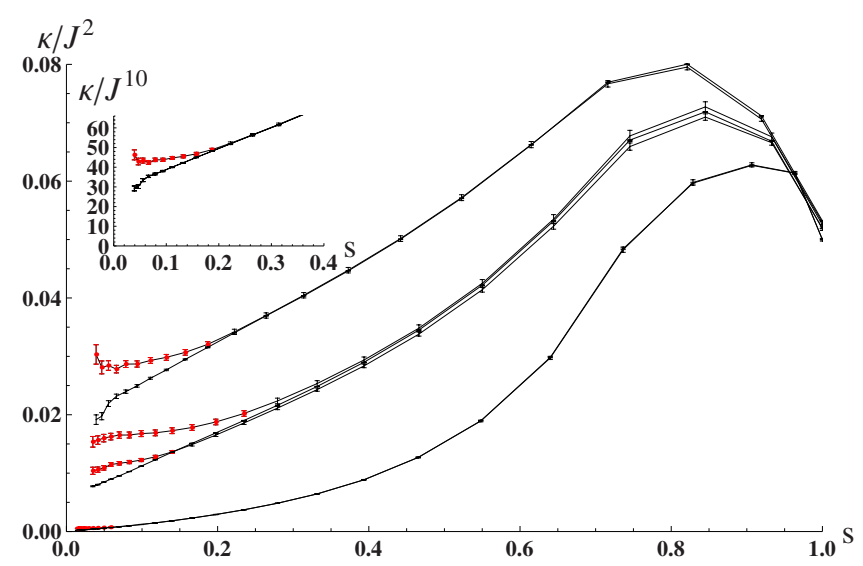

FIG. 8. (Color online) Finite-size effects for $J=0.16,0.32,0.40$ (bottom to top near $s=0$ ): 100 vs 20 spins for $J=0.40,10$, and 20 vs 100 spins for $J=0.32$ and 20 vs 100 spins for $J=0.16$. Red color is used to indicate the data influenced by finite-size effects according to Eq. (15) with $C_{D}=10$. Inset: $J=0.16$ data replotted.

not shown). So far we have not found a theoretical understanding of these interesting corrections to simple thermal diffusion.

\section{ACKNOWLEDGMENTS}

We thank J. Chalker, A. Dhar, S. Fishman, S. Girvin, J. Lebowitz, T. Spencer, and especially S. Sachdev for useful discussions. This work was supported by the NSF through Grant No. DMR-0603369 (V.O.) and MRSEC under Grant No. DMR-0819860 (D.A.H.). V.O. was also supported by Yale University. A.P. thanks the PCCM REU program at Princeton for support during the initial stages of this work.

\section{APPENDIX A: FINITE-SIZE EFFECTS}

We have checked that all of the extrapolations above are free from finite-size effects (by comparing against simulations on smaller and in some cases, larger samples). Nevertheless, it is interesting to consider the expected hydrodynamic size effects somewhat quantitatively, via Eq. (15). To illustrate this we display in Fig. 8 some results on shorter systems for $J=0.16,0.32,0.40$ that do show size effects: due to the periodic boundary conditions, the conductivity in smaller rings saturates in the dc limit at a value corresponding to the ac value at a "frequency" $2 \pi / t^{*}$ corresponding to $\cong C_{D} D(2 \pi / L)^{2}$, with $D \cong 3 \kappa$. Our results are qualitatively consistent this with $C_{D} \cong 10$ or slightly larger. It perhaps remarkable that despite orders of magnitude of variation in the diffusion constant in going from $J=0.4$ to 0.16 the crossover from bulk to finite system behavior is characterized by roughly the same constant $C_{D} \cong 10$.

\section{APPENDIX B: CHAOS AMPLIFICATION OF ROUNDOFF ERRORS}

No numerical study of a nonlinear classical dynamical system is complete without some understanding of the interplay of discretization and roundoff errors and chaos. We are 
studying a Hamiltonian system that conserves total energy, so the chaos is only within manifolds of constant total energy in configuration space. Thus although roundoff errors introduce tiny violations of energy conservation, these changes in the total energy are not subsequently amplified by the system's chaos; we have numerically checked that this is indeed the case. As a result of this precise energy conservation the energy transport computation remains well defined. The simulation is far less stable within an equal-energy manifold, where nearby trajectories diverge exponentially due to chaos. In particular, this means that the component of any roundoff error that is parallel to the equal-energy manifold is exponentially amplified by the chaos. At large $J$ this happens rather quickly while for small $J$ the chaos is weaker and longer individual trajectories can be retraced back to their respective initial conditions. However, at small $J$ very long runs are necessary to extrapolate to the dc thermal conductivity: in the end all of our extrapolations are done in the regime where all individual many-body trajectories are strongly perturbed by chaos-amplified roundoff errors.

Ultimately, however, we are only concerned with the stability of the current autocorrelations that enter in the Kubo formula for $\kappa$. Although the precise trajectories may diverge due to chaos-amplified roundoff errors, this need not have a strong effect on $C(t)$. To study this issue quantitatively we simulated roundoff noise of different strength in our computations. Specifically, we add extra random noise to the computation without altering the total energy by multiplying the angle each spin precesses in each time step by a factor of $1+\eta_{i}(t)$, where the $\eta_{i}(t)$ are independent random numbers uniformly distributed between $P$ and $-P$ ( $P=$ noise strength).

In 400 rings of 500 spins coupled with $J=0.14$ we simulated the same initial condition with different $\eta_{i}(t)$ and with different values of simulated noise $P=10^{0}, 10^{-1}$,

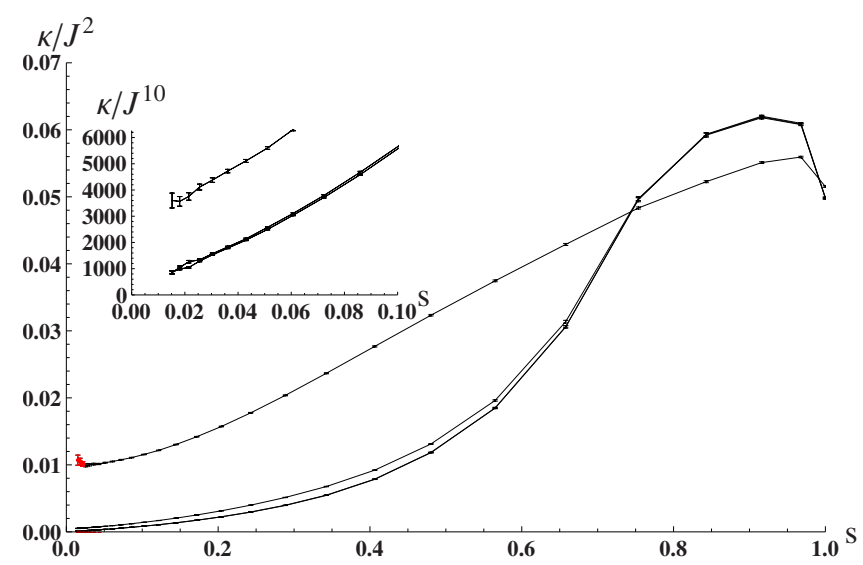

FIG. 9. (Color online) Simulated roundoff effects at $J=0.14$ : low frequency, long-time conductivity is largest for $P=1$, smaller for $P=0.1$, but essentially indistinguishable between $P=0$ and $P$ $=0.01$. Recall that intrinsic roundoff noise in our double-precision runs is $P_{\text {intr }} \lesssim 10^{-15}$. Inset: data with $P=0.1,0.01,0$.

$10^{-2}, 0$ - these results are presented in Fig. 9 below. As expected, the long-time insulating behavior is weakened by the presence of noise. Quantitatively, however, we observe little or no difference between results obtained in the presence of simulated noise with $P=10^{-2}$ vs ones obtained for intrinsic noise (which at double precision corresponds to $P_{\text {intr }}$ $\left.\lesssim 10^{-15}\right)$. Clearly, this statement heavily depends on the duration of the simulation, value of $J$, etc. Judging from Fig. 9 roundoff errors are not a serious source of uncertainty in our results in main text. Interestingly, it is also possible for strong noise to suppress $\kappa$, as indeed happens at shorter times, which can be traced here to a sort of "dephasing" of sharp response of quasiperiodic localized states.

\footnotetext{
*oganesyan@mail.csi.cuny.edu

†pal@princeton.edu

*huse@princeton.edu

${ }^{1}$ P. W. Anderson, Phys. Rev. 109, 1492 (1958).

${ }^{2}$ D. M. Basko, I. L. Aleiner, and B. L. Altshuler, Ann. Phys. 321, 1126 (2006).

${ }^{3}$ V. Oganesyan and D. A. Huse, Phys. Rev. B 75, 155111 (2007).
}

${ }^{4}$ S. Mukerjee, V. Oganesyan, and D. A. Huse, Phys. Rev. B 73, 035113 (2006).

${ }^{5}$ A. Dhar and J. L. Lebowitz, Phys. Rev. Lett. 100, 134301 (2008).

${ }^{6}$ R. W. Gerling and D. P. Landau, Phys. Rev. B 42, 8214 (1990).

${ }^{7}$ M. H. Ernst, J. Machta, J. R. Dorfman, and H. van Beijeren, J. Stat. Phys. 34, 477 (1984). 